\section{DIACRONIE}

\section{Diacronie}

Studi di Storia Contemporanea

$N^{\circ} 13,1 \mid 2013$

Contrabbandieri, pirati e frontiere: per una storia delle pratiche informali nell'America Centrale (XVII-XXI

secolo)

\title{
Unlawful enterprise
}

Il filibustering di Narciso López: people-to-people diplomacy tra schiavismo ed annessionismo

\section{Alessandro Badella}

\section{(2) OpenEdition}

\section{Journals}

Edizione digitale

URL: http://journals.openedition.org/diacronie/729

DOI: $10.4000 /$ diacronie. 729

ISSN: 2038-0925

Editore

Association culturelle Diacronie

Notizia bibliografica digitale

Alessandro Badella, «Unlawful enterprise », Diacronie [Online], № 13, 1 | 2013, documento 8, Messo

online il 01 avril 2013, consultato il 19 avril 2019. URL : http://journals.openedition.org/diacronie/729 ; DOI : 10.4000/diacronie.729 


\title{
Diacronie
}

N. 13 | 1|2013 Contrabbandieri, pirati e frontiere

8/

\section{Unlawful enterprise}

\section{Il filibustering di Narciso López: people-to-people diplomacy tra schiavismo ed annessionismo}

\author{
Alessandro BADELLA*
}

Le spedizioni clandestine di Narciso López rappresentarono uno dei primi contatti people-to-people a livello politico ed economico tra Cuba e gli Stati Uniti. Il diniego del supporto federale a tali iniziative illegali contro il dominio coloniale spagnolo a Cuba crearono un ampio movimento annessionista a New York così come a New Orleans e la attività di filibustering di López incarnarono una vera e propria risposta privata al fallimento della diplomazia nell'acquisizione di Cuba. L'epopea di López creò una solida joint venture cubano-americana volta a proteggere gli interessi creoli sull'isola nella preservazione del sistema schiavista, mentre molti uomini d'affari e imprenditori americani vedevano in Cuba un nuovo stato dell'Unione per espandere i propri interessi commerciali, incrementare la rappresentanza schiavista al Senato, fare fortuna con i Cuban bond o liberare l'isola dal dominio coloniale europeo.

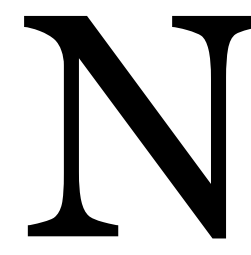
arciso López ebbe, alla metà del XIX secolo, una considerevole popolarità negli Stati Uniti così come sull'isola di Cuba, divenendo uno dei padri fondatori delle operazioni di filibustering. La nozione di filibustering oggi ha assunto un significato tipicamente politologico, trattandosi di una forma di ostruzionismo congressuale, attraverso l'uso di interventi prolungati, al fine di 
impedire alle proposte di legge di giungere alla fase della votazione ${ }^{1}$. Tuttavia, a metà dell'Ottocento, il termine aveva tutto'altro significato. Il filibuster, o filibustero, tra gli anni Quaranta e Sessanta dell'Ottocento, venne ad indicare un complesso fenomeno di spedizioni militari private verso paesi e ridotti coloniali europei contro cui il governo federale degli Stati Uniti non era formalmente in guerra. Nato come strumento di arricchimento o gloria personale con Francisco de Miranda e Aaron Burr, il filibustering, proprio alla metà dell'Ottocento, si sarebbe ammantato di nuovi significati ed obiettivi ${ }^{2}$. Nel giugno del 1850, nel pieno dell'epopea di López, un editoriale del «Delta» di New Orleans faceva notare come il termine filibuster avesse praticamente sostituito quella di "liberatore", fornendo quindi una nobile causa ad iniziative militari contro il dominio coloniale spagnolo sull'isola di Cuba³.

Narciso López nacque a Caracas nel 1797 e si formò militarmente nei ranghi realisti spagnoli, combattendo contro l'indipendenza delle colonie nelle battaglie di Queras del Medio e Carabobo. Dopo il ritiro delle truppe spagnole dell'America continentale, López si trasferì in Spagna, ove partecipò, col grado di colonello, alla prima guerra carlista a fianco dei liberali. Nel corso degli anni Trenta ricoprì il ruolo di rappresentante, per la città di Siviglia, alle Cortes, nonché quello di governatore militare di Valencia e Madrid, sino ad acquisire il grado di generale nel 1840. La sua permanenza in Spagna gli permise sia di assistere alla dissoluzione della rappresentanza dell'isola di Cuba all'interno delle strutture parlamentari della madrepatria, sia di venire a contatto con i circoli creoli liberali. Nel 1840, López si trasferì a Cuba al seguito del capitano generale Jerónimo Valdés che gli affidò la gestione delle province di Matanzas e Trinidad nonché la presidenza della commissione militare isolana. Dopo la rimozione di Valdés (1843), cadde politicamente in disgrazia e perse gran parte delle proprie fortune in avventure economiche disastrose. Politicamente López venne gradualmente attirato dalle forze creole annessioniste, come il Club de la Habana, e partecipò alla cospirazione del 1848 che, a causa di una

\footnotetext{
${ }^{1}$ Per una panoramica storica completa sull'ostruzionismo al Congresso cfr. COGER, Gregory, Filibustering: a political history of obstruction in the House and Senate, Chicago, University of Chicago Press, 2010.

${ }^{2}$ Sulle origini del filibustering cfr. BROWN, Charles H., Agents of Manifest Destiny. The Lives and Times of the Filibusters, Chapel Hill, University of North Carolina Press, 1980, pp. 3-18; MAY, Robert E., Manifest Destiny's Underworld: Filibustering in Antebellum America, Chapel Hill, University of North Carolina Press, 2002, pp. 1-18. Per una rassegna storica sull'evoluzione del fenomeno in riferimento all'espansionismo americano cfr. MAY, Robert E., Manifest Destiny's Filibusters, in HAYES, Sam W., MORRIS Christopher (eds.), Manifest Destiny and Empire: American Antebellum Expansionism, College Station, Texas A\&M University Press, 1997, pp. 146-178.

${ }^{3}$ Cit. in CHAFFIN, Tom, Fatal Glory. Narciso López and the First U.S. Clandestine War against Cuba, Charlottesville, University of Virginia Press, 1996, p. 1.
} 
delazione, fallì sul nascere. Ricercato dalle autorità coloniali spagnole, il generale venezuelano dovette riparare negli Stati Uniti come esule (luglio 1848).

Prima a New York e successivamente a New Orleans, López preparò le spedizioni private che avrebbero avuto il compito di liberare l'isola di Cuba dal dominio spagnolo. Già nell'agosto del 1849, López aveva avviato i preparativi per una invasione: nell'ottobre dello stesso anno le autorità americane bloccarono la spedizione del generale a Round Island. Il generale López ebbe modo di sbarcare due volte a Cuba, con l'intento di liberarla dal dominio coloniale spagnolo. La prima spedizione (maggio 1850), che invase e conquistò la città di Cárdenas (anche se solo per poche ore), venne respinta dalle forze spagnole. Nell'estate dell'anno seguente, ne organizzò una seconda, che si concluse con un fallimento completo: gli uomini della missione vennero catturati e molti di essi subirono la sorte del proprio comandante, condannato a morte tramite garrota il $1^{\mathrm{o}}$ settembre $1851^{4}$.

Celebrato da alcuni come un autentico indipendentista, privo di ogni velleità di gloria o arricchimento personale e lontano da ogni mira annessionista, un «leader per natura» o, al contrario, denigrato come «quella canaglia di López» e dipinto come un «traditore della propria patria anziché un difensore»" è indubbio che Narciso López, oggi quasi dimenticato, sia stato un personaggio complesso ma fondamentale nella costruzione delle relazioni cubano-statunitensi ed autentico simbolo dei differenti interessi (settari e non) che mettevano Cuba e le sue vicende interne al centro delle preoccupazioni di Washington, così come avrebbero dimostrato la Guerra CubanoIspano-Americana e la costituzione della República plattista.

Gli interessi schiavisti e commerciali degli Stati Uniti sull'isola di Cuba, la formazione tra le due sponde dello Stretto di Florida di metà Ottocento di una peopleto-people diplomacy e l'attività rivoluzionaria degli esuli cubani nelle città di New York e New Orleans, generarono un vasto movimento in favore della liberazione di Cuba dall'oppressione coloniale spagnola; che poteva essere condotta solamente attraverso una forma di aiuto privato dall'esterno (ovvero dagli Stati Uniti), come testimonia

\footnotetext{
${ }^{4}$ Per un account cronologico sulle spedizioni militare di López cfr. CHAFFIN, Tom, op. cit.; PORTELL VILÁ, Herminio, Narciso López y su época, 3 voll., La Habana, Cultural, 1930; CALDWELL, Robert G., The Lopez Expeditions to Cuba, 1848-1851, Princeton, Princeton University Press, 1915; BROWN, Charles H., op. cit., pp. 67-88.

${ }^{5}$ Cfr. PORTELL VILÁ, Herminio, op.cit., vol. 1, pp. 185-192; QUISENBERRY, Anderson C., Lopez's Expeditions to Cuba, 1850 and 1851, Louisville, Morton \& Co., 1906, p. 28; Lettera di Gilman A. Cook (membro dell'ultima spedizione di López), 16 agosto, 1851 in JONES, Alexander, Cuba in 1851, New York, Stringer and Townsend, 1851; Lettera di James St. Levi, in WILSON, Thomas W., An Authentic Narrative of the Piratical Descents upon Cuba, La Habana, s.e., 1851, p. 37. In molte lettere di commiato inviate a familiari e parenti dai condannati a morte dell'ultima spedizione si evince la delusione per il fallimento dell'impresa, imputato alla scarsa capacità militare del generale López.
} 
l'epopea del flibustering contro la colonia cubana. L'epopea di Narciso López fornisce un interessante spaccato sulle reti di relazione che si andarono sviluppando tra Cuba e Stati Uniti. La stessa natura privata delle imprese di filibustering di López attribuì un ruolo di primissimo piano ai contatti informali che il generale venezuelano ebbe modo di costituire nel corso della sua permanenza sull'isola e negli Stati Uniti. Nel 1848, López partecipò all'organizzazione di una cospirazione anti-coloniale, la Conspiración de la Mina de la Rosa Cubana, nella quale vennero coinvolti alcuni dei principali membri del futuro Club de La Habana (di idee annessioniste), ma anche alcuni cospiratori, come José María Sánchez Iznaga, che successivamente aiutarono López nella preparazione delle spedizioni militari dagli Stati Uniti ${ }^{6}$.

Narciso López, trasferitosi negli Stati Uniti, venne a contatto direttamente con la realtà cubana lì presente e con le reti formatesi dai primi anni Venti grazie a José María Heredia e Félix Varela7. La comunità cubano-americana dei primi decenni dell'Ottocento era sì ridotta nel numero (inferiore alle mille unità), ma con un'istruzione molto elevata: i primi nuclei di esuli cubani erano formati da liberi professionisti, avvocati, possidenti e studenti ${ }^{8}$. La costruzione di reti informali tra Cuba ed alcuni centri decisionali europei e statunitensi era già stata brevemente sperimentata durante il governo liberale spagnolo: una serie di intellettuali cubani di matrice liberale avevano utilizzato importanti canali letterari, come la Comisión permanente de literatura, per avanzare richieste politiche del sistema di rappresentanza all'interno delle Cortes peninsulari ${ }^{9}$. Se nei primi due decenni dell'Ottocento le forme di pressione informale si rivolgevano alla Madrepatria, nel corso degli anni Quaranta, tali sforzi organizzativi si spostarono nettamente verso gli Stati Uniti.

Le cospirazioni cubane del 1847 e 1848 vennero organizzate e finanziate da una società di orientamento annessionista, il Club de La Habana, composto da Miguel

\footnotetext{
${ }^{6}$ Cfr. MESA RODRÍGUEZ, Manuel I., «La Conspiración de la Mina de la Rosa Cubana», in Cuadernos de Historia Habanera, 44, 1950, pp. 35-58.

${ }^{7}$ Circa le prime organizzazioni cubano-americane negli Stati Uniti cfr. MCCADDEN, Joseph J., «The New York-to-Cuba Axis of Father Varela», in The Americas, 20, 4/1964, pp. 376-392; GRUESZ, Kristen Silva, Ambassador of Culture: the Transamerican Origins of Latino Writing, Princeton, Princeton University Press, 2002, pp. 30-48; MCCADDEN, Joseph, MCCADDEN, Helen, Father Varela. Torch Bearer from Cuba, New York, U.S. Catholic Historical Society, 1969. Sulla migrazione di intellettuali cubani verso gli Stati Uniti nel periodo del filibustering di López cfr. GAY CALBO, Enrique, «Los emigrados cubanos en los Estados Unidos (1848-1849)», in Cuadernos de Historia Habanera, 44, 1950, pp. 81-95.

${ }^{8}$ Cfr. POYO, Gerald E., With All, and for the Good of All: The Emergence of Popular Nationalism in the Cuban Communities of the United States, 1848-1898, Durham, Duke University Press, 1989, pp. 2-4, 9-11.

${ }^{9}$ Cfr. AGUILERA MANZANO, José, «The Informal Communication Network Built by Domingo del Monte from Havana between 1824 and 1845», in Caribbean Studies, 37, 1/2009, pp. 67-96.
} 
Aldama, Manuel Rodríguez Mena, Domingo Goicuría, José Antonio Echeverría e José Luis Alfonso, che si fecero interpreti delle preoccupazioni dei piantatori bianchi dell'isola. Il Club isolano venne costituito sul modello del Concilio Cubano di New York con Gaspar Betancourt Cisneros e Cristóbal Madán come trait d’union tra gli Stati Uniti e l’isola. Peraltro Madán stava operando da alcuni anni negli Stati Uniti attraverso il Consejo de Gobierno Cubano ${ }^{10}$. Nello stesso periodo, negli Stati Uniti venne ad operare il Consejo Cubano ${ }^{11}$. Alcuni futuri dirigenti del Club, come José Luis Alfonso e Miguel Aldama, già nel 1847, ospitarono a Cuba il direttore del «Sun» di New York, Moses Yale Beach, ed il giornalista O'Sullivan, che si riveleranno, negli anni successivi, preziosi alleati della causa annessionista e delle imprese di López. L’incontro tra O’Sullivan e Beach e l'inizio della loro attività di lobby a favore dell'annessione di Cuba possono fornire una misura della dimensione assolutamente privata e people-to-people di questa prima fase dei rapporti cubano-americani. Al momento della riunione con il Club (gennaio 1847), O’Sullivan, cognato di Madán, si trovava a Cuba in viaggio di nozze, mentre Beach stava prendendo informazioni per l'editoriale dal titolo Cuba under the Flag of the Unites States, che sarebbe uscito sul «Sun» il 23 luglio dello stesso anno ${ }^{12}$.

Già dal 1847, gli esuli cubani stabilitisi a New Orleans si riunivano attorno al quotidiano «La Patria», mentre un immigrato cubano, José M. Vingut, aveva fondato a Washington il giornale «La Aurora», che propagandava le proposte annessioniste del senatore Levy Yulee ${ }^{13}$. Nel 1848, negli Stati Uniti venne fondato il quotidiano «La Verdad», che, stampato presso la sede del "Sun" diretto da Beach, iniziò le proprie pubblicazioni l'8 gennaio 1848 grazie al contributo iniziale di 10 mila dollari provenienti da influenti circoli annessionisti cubani. Sino al 1860, anno di chiusura del periodico, esso circolò gratuitamente negli Stati Uniti e (illegalmente) anche a Cuba ${ }^{14}$. Dalla metà degli anni Quaranta, le connessioni cubane con alcuni membri dell'apparato congressuale-militare erano già strutturate. Dal 1845 vi erano state iniziative di membri

\footnotetext{
${ }^{10}$ Cfr. PÉREZ, Louis A., Cuba and the United States: Ties of Singular Intimacy, Athens, Georgia University Press, 2003, pp. 45-46.

${ }^{11}$ Per una panoramica sul ruolo rivoluzionario ed annessionista del Club cfr. PICHARDO, Hortensia, «El Club de La Habana an la conspiraciones de la época», in Cuadernos de Historia Habanera, 44, 1950, pp. 59-80.

${ }^{12}$ Cfr. CHAFFIN, Tom, op. cit., pp. 13, 22-23.

${ }^{13}$ Cfr. ZARAGOZA, Justo, Las insurrecciones en Cuba, vol. 1, Madrid, Imprenta Miguel G. Hernández, 1872, pp. 565-569.

${ }^{14}$ Cfr. RODRÍGUEZ, José Ignacio, Estudio histórico sobre el origen, desenvolvimiento y manifestaciones prácticas de la idea de la anexión de la Isla del Cuba a los Estados Unidos de América, La Habana, La Propaganda Literaria, 1900, p. 108; LAZO, Rodrigo, Writing to Cuba: filibustering and Cuban exiles in the United States, Chapel Hill, University of North Carolina Press, 2005, pp. 74-76; GONZÁLEZ, Juan, TORRES, Joseph, News for All the People: The Epic Story of Race and the American Media, London, Verso Books, pp. 78-81.
} 
del Congresso per avanzare alla Spagna una concreta proposta di acquisto dell'isola ${ }^{15}$. Peraltro, si trattava di correnti politiche congressuali che operavano alla luce del sole per propugnare la causa dell'annessione di Cuba agli Stati Uniti, tanto che il ministro spagnolo a Washington, in una missiva dell'agosto 1850 al Segretario di Stato Webster (in relazione al primo sbarco di Narciso López), ebbe modo di elencare con precisione alcune di queste proposte, tra cui quella del senatore della Florida David Levy Yulee ${ }^{16}$. Inoltre, fu proprio il Club a contattare, nel 1848, il generale statunitense William Worth (deceduto a causa del colera nel maggio del 1849) ed un loro emissario, José Ambrosio Gonzáles, fu incaricato di intercettare López per proporgli la guida di una spedizione per la liberazione di Cuba ed un finanziamento di 30.000 dollari (giugno 1849) ${ }^{17}$.

Spinto dagli attriti personali con alcuni membri del Club e del Consejo, López fu in grado di sviluppare un'attività autonoma volta alla preparazione delle attività di filibustering: nel dicembre del 1849, pochi mesi dopo il fallimento della prima spedizione verso Cuba, il generale cubano fondò una nuova organizzazione che prese il nome di Junta Promovedora de los Intereses Políticos de Cuba. La Junta, nella quale confluirono anche José Ambrosio Gonzáles e lo scrittore Cirilo Villaverde, fu in grado di mantenere ed espandere i propri contatti e le fonti di finanziamento ${ }^{18}$. Infatti, nonostante dal febbraio 1850 tutte le sovvenzioni provenienti dal Club si fossero interrotte, López poté proseguire la preparazione di nuove imprese proprio grazie a contatti personali (o mediati) con esponenti della classe dirigente newyorkese e sudista, data la grande popolarità di cui godeva il generale cubano. Nel dicembre del 1849, Gonzáles e López vennero avvicinati dall'avvocato ed ex senatore John Henderson che, presentatosi come «un amico della causa cubana», consigliò di spostare la base operativa da New York a New Orleans per sfruttare un ambiente proficuo per le attività in cui López era impegnato ${ }^{19}$. Inoltre, fu proprio il giornalista O’Sullivan, che da New York mantenne i contatti con gli esuli cubani affiliati a López, a procurare l'imbarcazione "Cleopatra" utilizzata dal generale cubano per l'ultima tragica spedizione (aprile 1851). O'Sullivan fu un prezioso alleato della causa cubana e del filibustering di López, considerando l'accesso diretto che godeva presso il presidente

${ }^{15}$ Cfr. MORALES Y MORALES, Vidal, Iniciadores y primeros martires de la revolución cubana, vol. 2, La Habana, Cultural, 1931, pp. 565-569.

${ }^{16}$ Cfr. Lettera di Calderón de la Barca a Daniel Webster, 2 agosto, 1850 in CALDWELL, Robert Granville (ed.), Correspondence on the Lopez Expedition to Cuba, 1849-51, United States National Archives, Record Group 59, General Records of the State Department, Miscellaneous Correspondence 1784-1906, pp. 20-21.

${ }^{17}$ Cfr. PORTELL VILÁ, Herminio, op. cit., vol. 1, p. 235.

${ }_{18}^{18}$ RAUCH, Basil, American Interest in Cuba, 1848-1955, New York, Octagon, 1974, p. 109.

${ }^{19}$ Cfr. CHAFFIN, Tom, op. cit., p. 82. 
Polk²0. Il 10 maggio del 1848, O’Sullivan ed il senatore S. Douglass dell'Illinois intercedettero presso la Casa Bianca per proporre un intervento americano per l'annessione di Cuba. Polk, nonostante non avesse una particolare posizione sull'argomento, si disse «ardentemente favorevole ad acquistare Cuba per fare di essa uno stato dell'Unione», salvo però mostrarsi contrario ad una spedizione militare ${ }^{21}$. Inoltre, il giornalista O'Sullivan, che venne accusato di violazione del Neutrality Act nel 1851, utilizzò i propri canali politico-istituzionali per eludere la condanna: illustri avvocati e uomini politici newyorkesi, come David Dudley Field e Samuel Tilden, furono chiamati al banco dei testimoni per difenderlo dalle accuse.

José Ambrosio Gonzáles fu il principale agente cubano nell'ultima fase dell'epopea di López, soprattutto dopo il trasferimento della base operativa a New Orleans. Il fatto che López non conoscesse la lingua inglese e quindi non fosse in grado di comunicare direttamente con gli interlocutori statunitensi, poneva al centro della macchina organizzativa della Junta proprio Gonzáles. Nato a Matanzas nel 1818, aveva trascorso un periodo di studi negli Stati Uniti, ove si era stabilito dalla fine degli anni Quaranta, apportando alla causa cubana una serie innumerevole di sostenitori e potenziali finanziatori. Gonzáles fu anche il principale fornitore dei contatti che servirono a López per ricevere assistenza legale durante il biennio 1850-1851 ed ottenere una serie di importanti colloqui con personalità di spicco dell'annessionismo schiavista, come John Quitman, Robert Lee e Jefferson Davis. Anche dopo la morte di López, Gonzáles avrebbe mantenuto i contatti con importanti membri dell'aristocrazia del Sud (soprattutto dopo il matrimonio con la figlia di un ricco piantatore della Carolina), sia in qualità di organizzatore delle ultime imprese private per liberare l'isola, sia come membro effettivo dell'esercito confederato durante la Guerra Civile ${ }^{22}$.

Un ulteriore elemento essenziale per l'analisi dell'esperienza organizzativa del filibustering di López, che ha sottolineato De la Cova, è quello dell'affiliazione

\footnotetext{
${ }^{20}$ Sulla figura di O'Sullivan, il cui padre morì in una spedizione di filibustering in Venezuela, nel rapporto con il mondo politico ed economico statunitense cfr. WIDMER Edward L., Young America: The Flowering of Democracy in New York City, New York, Oxford University Press, 1999, pp. 185-187. Per una biografia cfr. SAMPSON, Robert D., John L. O'Sullivan and His Times, Kent, Kent State University Press, 2003.

${ }^{21}$ Cfr. QUAIFE, Milo M. (ed.), The Diary of James K. Polk during his Presidency, 1845 to 1849, 3 voll., Chicago, McClurg, 1910, pp. 446, 485-487 ed anche BOTERO, Rodrigo, Ambivalent Embrace: America's Troubled Relations with Spain from the Revolutionary War to the Cold War, Westport, Greenwood Press, 2001, pp. 71-73.

${ }^{22}$ Cfr. DE LA COVA, Antonio R., Cuban Confederate colonel: the life of Ambrosio José Gonzáles, Columbia, University of South Carolina, 2003, capp. 2-3; JONES, Lewis P., «Ambrosio José Gonzáles, a Cuban Patriot in Carolina», in The South Carolina Historical Magazine, 56, 2/1955, pp. 67-76; STEVENS, Michel W., Two Falgs, One Cause - A Cuban Patriot in Gray: Ambrosio José Gonzáles, in TUCKER, Philip T. (ed.), Cubans in the Confederacy, Jefferson, McFarland, 2002, pp. 143-223.
} 
massonica. Le logge massoniche americane fornirono il supporto logistico a López e compagni, facilitando le relazioni con l'establishment politico, con alcuni membri del sistema giudiziario statunitense e nella raccolta dei fondi e degli armamenti per le spedizioni. In sostanza, la fratellanza massonica, che coinvolse anche López e i suoi seguaci, fornì un elemento di facilitazione del filibustering, un quid pluris che ne avrebbe reso possibile la realizzazione ${ }^{23}$.

La fitta rete di contatti personali permise agli esuli cubani di eludere buona parte della sorveglianza delle autorità giudiziarie e marittime preposte ad evitare le spedizioni private contro Cuba. I presidenti Zachary Taylor e Millard Fillmore, successori di Polk, si opposero alle spedizioni di López sul territorio cubano, cercando di promuovere il rispetto dei trattati con la Spagna e applicando il Neutrality Act, che puniva i responsabili di azioni ostili nei confronti di paesi con i quali gli Stati Uniti non fossero direttamente in conflitto ${ }^{24}$. Nei confronti di Cuba, l'attendismo politico della presidenza era volto ad appoggiare il dominio coloniale spagnolo, nel tentativo di prevenire un intervento inglese senza però precludersi la possibilità di una annessione sul modello texano, fiduciosi che una indipendenza cubana avrebbe portato l'isola a "gravitare" naturalmente verso l'Unione25. Risulta evidente che senza una fitta rete di contatti e appoggi privati di personaggi di spicco della politica e della società

\footnotetext{
${ }^{23}$ Cfr. DE LA COVA, Antonio Rafael, «Filibusters and Freemasons: The Sworn Obligation», in Journal of the Early Republic, 17, 1/1997, pp. 106-110.

${ }^{24}$ Cfr. LANGLEY, Lester D., "The Whigs and the López Expeditions to Cuba, 1849-1851: A Chapter in Frustrating Diplomacy», in Revista de Historia de América, 71, 1971, pp. 9-11; TORRENTE, Mariano, Bosquejo económico político de la Isla de Cuba, Madrid, Impr. de M. Pita, 1852, pp. 60-65; BAUER, Jack K., Zachary Taylor: soldier, planter, statesman of the old Southwest, Baton Rouge, Louisiana State University Press, 1985, pp. 277-279; CHAMBERLAIN, Ivory, FOOTE, Thomas M., Biography of Millard Fillmore, Buffalo, Thomas \& Lathrops, 1856, p. 186; CHAFFIN, Tom, op. cit., pp. 168-169.

${ }^{25}$ Sul ruolo del sistema internazionale nella politica estera americana verso Cuba, sino al 1850, cfr. CALLAHAN, James M., Cuba and International Relations, Baltimora, Johns Hopkins Press, 1899; MULLER, Dalia A., "Latin America and the Question of Cuban Independence», in The Americas, 68, 2/2011, pp. 209-239; PÉREZ, Louis A., Cuba and the United States: Ties of Singular Intimacy, cit., pp. 1-54; BADELLA, Alessandro, La spina nel fianco. Stati Uniti e Cuba in una prospettiva razziale, 1823-1912, Salerno, Arcoiris, 2012, pp. 66-90; per una panoramica storica sull'effetto della "dottrina Monroe" sul futuro politico di Cuba cfr. DENT, David W., The Legacy of the Monroe Doctrine: A Reference Guide to U.S. Involvement in Latin America and the Caribbean, Westport, Greenwood Press, pp. 116-118; GILDERHUS, Mark T., «The Monroe Doctrine: Meanings and Implications», in Presidential Studies Quarterly, 36, 1/2006, pp. 5-16; GLEIJESES, Piero, «The Limits of Sympathy: The United States and the Independence of Spanish America», in Journal of Latin American Studies, 24, 3/1992, pp. 481-505; una descrizione del "gioco texano" degli Stati Uniti nei confronti di Cuba è contenuta in MADDEN, Richard R., The island of Cuba, London, C. Gilpin, 1849, pp. 85-87. Sulla "gravitazione" internazionale di Cuba cfr. Lettera a Hugh Nelson, 28 aprile, 1823, in FORD, Worthington C., The Writing of John Quincy Adams, vol. 7, New York, MacMillan, 1917, pp. 370-374 ed anche BEMIS, Samuel F., John Quincy Adams and the Foundation of American Foreign Policy, New York, Alfred A. Knopf, 1949.
} 
americana, sarebbe stato praticamente impossibile provvedere all'organizzazione di imprese di filibustering che presentavano pubblicamente i propri progetti.

Tuttavia, al di là degli influenti contatti personali di López a Washington così come nel Sud, le iniziative private contro Cuba ebbero un grande rilievo presso l'opinione pubblica degli Stati Uniti. Frequenti furono i meeting pubblici a sostegno del generale venezuelano, pubblici interventi di simpatizzanti e sostenitori organizzati attraverso una rete capillare. Le manifestazioni pubbliche di apprezzamento alla causa cubana e al filibustering continuarono anche dopo la morte di López, tanto che dopo il fallimento dell'ultima spedizione ne vennero organizzate almeno venticinque, animate dal revanscismo contro le autorità spagnole che, oltre López, avevano giustiziato anche alcuni cittadini americani ${ }^{26}$. La stessa mitizzazione della figura del generale cubano presso l'opinione pubblica sopravvisse alla sua morte, stimolando la produzione artistica di una generazione di esuli cubani e contribuendo alla mitizzazione della figura del filibustero ${ }^{27}$.

Data l'ambiguità del disegno post-coloniale di López, le sue imprese guadagnarono sostegno anche nelle città di New York, Baltimora e Philadelphia da parte di intellettuali e gente comune (celeberrima fu la manifestazione in onore di López tenutasi a New York nell'agosto 1851, a cui parteciparono oltre 8.000 persone) affascinati dalla possibilità di "convertire" Cuba agli ideali libertari statunitensi ${ }^{28}$ Gli interventi pubblici avevano lo scopo di reclutare adepti, ma anche quello di finanziare le campagne militari attraverso la raccolta fondi e l'acquisto di Cuban bonds, che divennero una delle principali sorgenti di denaro, soprattutto dopo la rottura con il Club e il Concilio ${ }^{29}$. La possibilità di arricchimento da parte dei membri delle spedizioni fu una delle cause dell'interesse popolare verso le imprese di López. Come scriveva il capitano J.C. Davis della Louisiana, la sete di gloria e di oro aveva spinto molti giovani avventurieri americani a lanciarsi in quella che prometteva essere una «piccola operazione per l'estate», un diversivo estivo per fare fortuna e ritornare negli Stati Uniti prima dell'autunno ${ }^{30}$. Nel 1849 ai partecipanti alla spedizione erano stati promessi 7 dollari al giorno, più un bonus finale di 4.000, mentre vennero emessi bond per oltre 2

${ }^{26}$ Sul ruolo delle manifestazioni pubbliche a sostegno del filibustering di López cfr. GREENBERG, Amy S., Manifest Manhood and the Antebellum American Empire, Cambridge, Cambridge University Press, 2005, pp. 183-190.

${ }^{27}$ Cfr. LAZO, Rodrigo, Writing to Cuba., cit., pp. 108-124.

28 Cfr. CHAFFIN, Tom, «Sons of Washington: Narciso López, Filibustering, and U.S. Nationalism, 1848-1851», in Journal of the Early Republic, 15, 1/1995, pp. 88-90.

${ }^{29}$ Sulla vendita dei bond cfr. CALDWELL, Robert G., op . cit., p. 44-46, 89; CHAFFIN, Tom, op. cit., p. 73, 90-91; MORALES Y MORALES, Vidal, op. cit., vol. 2, pp. 194-195.

${ }^{30}$ O.D.D.O. (pseud.), The History of the Late Expedition to Cuba, New Orleans, Daily Delta, 1850, pp. 3-4. 
milioni di dollari con una rendita del 6 per cento, che attirarono ricchi commercianti e speculatori anche a Washington e New York ${ }^{31}$.

Lo spostamento della Junta a New Orleans determinò un reclutamento massiccio tra i generali e le milizie degli Stati del Sud. La città rappresentava, già dall'epopea texana della metà degli anni Trenta, una la base operativa del filibustering statunitense contro i messicani del Texas e la New Orleans Association, che riuniva uomini d'affari e possidenti locali, aveva finanziato generosamente tali iniziative e l'opinione pubblica si presentava particolarmente ricettiva e sensibile a tali imprese militari32. Il tour di López e Gonzáles nel Sud (1850) fu in grado di costituire tre reggimenti con uomini provenienti da Louisiana, Kentucky e Mississippi, attingendo anche ai fondi dell'editore del «Delta» di New Orleans (anch'egli esule cubano con simpatie annessioniste)33. A New Orleans le imprese di López riuscirono a coniugare lo spirito di avventura di giovani ed intraprendenti statunitensi con l'espansionismo schiavista, le rivendicazioni nazionali dell'Europa continentale del 1848 (alla spedizione del 1851 parteciparono diversi austro-ungheresi reduci dai falliti moti continentali), la leyenda negra antispagnola, la presunta superiorità anglosassone ed il mito eroico del cittadino-soldato, resuscitato dalla guerra contro il Messico34. Se nel 1849, anche a causa della scarsità di fondi, la spedizione di Round Island fu abortita sul nascere, le successive imprese riuscirono a sbarcare a Cuba (salvo poi fallire tragicamente sul suolo cubano) anche grazie al grande supporto economico-politico delle élites del Sud35. Inoltre, l’ingresso nell'Unione della California come stato non schiavista (1850) aveva dato avvio ad un interessamento crescente del Sud per l'estensione della schiavitù anche a territori al di fuori del territorio continentale nordamericano, al fine di riequilibrare i rapporti di forza con il Nord all'interno del Senato.

Sebbene López non rappresentasse univocamente uno strumento nelle mani dell'espansionismo sudista e quindi non fosse interamente compromesso con la preservazione della schiavitù sull'isola di Cuba ed annessione agli Stati Uniti, risulta evidente che buona parte dei finanziatori e sostenitori delle sue attività di "pirateria" lo

\footnotetext{
${ }^{31}$ BROWN, Charles H., op. cit., pp. 55-56; CHAFFIN, Tom, op. cit., pp. 82-84.

${ }^{32}$ Cfr. MILLER, Edward L., New Orleans and the Texas Revolution, College Station, Texas A\&M University Press, 2004, pp. 24-26, 62, 103 ed anche TANSEY, Richard, «Southern Expansionism: Urban Interests in the Cuban Filibusters», in Plantation Society, 1-2/1979, pp. 227-251.

${ }^{33}$ Cfr. QUISENBERRY, Anderson C., op. cit., pp. 33, 66-68.

${ }^{34}$ Cfr. CHAFFIN, Tom, op. cit., p. 111. Circa il pregiudizio anti-spagnolo nelle imprese di López cfr. MAY, Robert E., "Young American Males and Filibustering in the Age of Manifest Destiny: The United States Army as a Cultural Mirror», in Journal of American History, 78, 1991, pp. 857-886, 862; LAZO, Rodrigo, Writing to Cuba, cit., pp. 85-91.

${ }^{35}$ Cfr. PORTELL VILÁ, Herminio, op. cit., vol. 2, p. 190.
} 
fossero. Infatti, anche Portell Vilá, sebbene abbia fornito l'interpretazione più indipendentista di López, ha ammesso il suo cedimento ai settori annessionisti e schiavisti al fine di attenerne l'appoggio politico, militare ma soprattutto economico ${ }^{36}$. Gran parte dei contatti americani di López erano propensi ad una annessione. Il Club de la Habana così come le associazioni cubano-americane degli anni Quaranta avevano una matrice formalmente annessionista e, dal 1850, questi ultimi avevano esautorato López dal comando delle spedizioni da loro finanziate proprio perché non avevano ottenuto garanzie di una attività militare diretta univocamente verso l'incorporazione negli Stati Uniti. Un articolo pubblicato da Cirilo Villaverde su «Democratic Review» nel febbario del 1850 sosteneva che l'obiettivo principale di López fu, sin dall'inizio, l'indipendenza di Cuba e la sua annessione agli Stati Uniti37.

Verso la fine degli anni Quaranta, la penetrazione commerciale statunitense era tanto significativa da soppiantare il commercio dell'isola con la madrepatria e con gli altri paesi europei. Nel 1848, il numero delle navi americane che commerciavano con Cuba era il doppio rispetto a quelle spagnole e molti cittadini dell'Unione possedevano imprese agricole e di trasporto. Non a caso, il primo tratto ferroviario sull'isola, inaugurato nel 1837, fu costruito da un ingegnere statunitense (anche se con capitale inglese) ${ }^{38}$. Buona parte del benessere isolano era strettamente legato al proficuo commercio con gli Stati Uniti, che contribuirono alla meccanizzazione della produzione agricola tra gli anni Quaranta e Cinquanta. Circa 400 macchinisti statunitensi, lavoratori stagionali specializzati nella raccolta meccanica della canna da zucchero, raggiungevano l’isola ogni anno e rappresentavano «il migliore strumento di propaganda per le idee annessioniste»39. Richard Madden, in un resoconto del 1849 sulla propria esperienza di sovrintendente alla liberazione degli schiavi africani a Cuba, scriveva: «Cuba, sin da quando la conosco, sta diventando lentamente, ma progressivamente, americanizzata. [...] Nel corso degli ultimi sette anni, l'opinione favorevole all'indipendenza, ampiamente diffusa all'interno della colonia, si è mutata in un desiderio di annessione agli Stati Uniti»40. Solo pochi anni più tardi, lo scrittore inglese Anthony Trollope avrebbe ricavato la stessa impressione ${ }^{41}$.

\footnotetext{
${ }^{36}$ Cfr. PORTELL VILÁ, Herminio, op. cit., vol. 2, pp. 186-190.

${ }^{37}$ Cfr. «General López, the Cuban Patriot», in United States Magazine and Democratic Review, 25/1850, pp. 97-112.

${ }^{38}$ JONES, Alexander, op. cit., p. 15.

${ }^{39}$ Cfr. DE LA CONCHA, José, Memorias sobre el estado político, gobierno y administración de la Isla de Cuba, Madrid, Impr. José Trujillo, 1853., p. 244.

${ }^{40}$ Cfr. MADDEN, Richard R., op. cit., p. 54 .

${ }^{41}$ Cfr. TROLLOPE, Anthony, The West Indies and the Spanish Main, Londra, Chapman \& Hall, 1860, p. 136. L'autore britannico osservava come il commercio isolano fosse precipitato nelle
} 
Anche i primi contatti culturali statunitensi con la produzione letteraria ed intellettuale isolana, strutturatisi nella prima metà dell'Ottocento, manifestarono legami molto profondi con l'agenda politica espansionista e del Manifest Destiny. Nel 1840, Alexander Hill Everett, diplomatico e uomo di lettere di Boston, venne inviato dal presidente Van Buren a Cuba ove conobbe Domingo Del Monte, intellettuale cubano che sarebbe stato accusato di aver fomentato l'insurrezione nera della metà degli anni Quaranta. Everett, attraverso un fitto scambio epistolare con Del Monte, raccolse informazioni sullo stato politico-sociale dell'isola; le avrebbe successivamente utilizzate per la pubblicazione di articoli di stampo annessionista su «North American Review» e su «Democratic Review» di O’Sullivan. Quest'ultimo, celeberrimo coniatore del "Destino manifesto", ospitava nelle pagine della propria rivista anche gli scritti di Cirilo Villaverde ugualmente impegnato nell'inclusione di Cuba nell'Unione42. Molti intellettuali ed attivisti vicini a López vedevano negli Stati Uniti un approdo sicuro per sfuggire alla censura e alla persecuzione delle autorità spagnole. Secondo la comunità cubano-americana, tale contatto intellettuale avrebbe potuto creare un legame profondo con l'opinione pubblica statunitense, una people-to-people diplomacy in grado di influenzare il decision making internazionale degli Stati Uniti43. Occorre ricordare anche la produzione giornalistica di Jane Cazneau, che pubblicò per il «Sun» e «La Verdad» con lo pseudonimo di “Cora Montgomery”, impegnata ad intercedere presso il presidente Polk per l'acquisto dell'isola. Anche Jane Cazneau, che assieme al marito William Cazneau sarebbe stata impegnata nell'attività di lobby finalizzata all'espansione commerciale in America Centrale, contribuì a mettere in contatto gli esuli cubani con l'opinione pubblica statunitense, cercando di contemperare i timori del Nord circa l'avanzata dello schiavismo sudista verso il bacino caraibico 44 .

Nel corso dell'Ottocento, gli Stati Uniti ebbero modo di "stregare" una serie considerevole di intellettuali latinoamericani e l'esperienza del viaggio in Nord America divenne una pratica particolarmente diffusa nella formazione cultural-politica delle

mani degli stranieri, soprattutto americani, ipotizzando che La Habana, di lì a poco, sarebbe potuta divenire una nuova New Orleans.

${ }^{42}$ Cfr. BRICKHOUSE, Anna, Transamerican Literary Relations and the Nineteenth-Century Public Sphere, Cambridge, Cambridge University Press, 2004, pp. 160-173.

${ }^{43}$ Cfr. LAZO, Rodrigo, "A Man of Action": Cirilo Villaverde as Trans-American Revolutionary Writer, in HERRERA-SOBEK, María, SÁNCHEZ KORROL, Virginia, Recovering the U.S. Hispanic Literary Heritage, Houston, Arte Público Press, 2000, pp. 319-321.

${ }^{44}$ Sul ruolo diplomatico informale dei coniugi Cazneau nell'espansionismo americano cfr. MAY, Robert E., «Lobbist for Commercial Empire: Jane Cazneau, William Cazneau, and U.S. Caribbean Policy, 1846-1878», in Pacific Historical Review, 48, 3/1979, pp. 383-412. 
élites dell'America meridionale, Cuba inclusa 45 . Non è un caso che le autorità spagnole vedessero gli studi negli Stati Uniti dei rampolli dell'aristocrazia cubana come uno dei principali vettori di instabilità politica, proprio perché fornivano ai creoli isolani gli strumenti per "esportare" il dibattito circa il futuro politico della colonia ${ }^{46}$. A Cuba, proprio alla vigilia delle spedizioni di López, l'opera Ideas sobre la incorporación de Cuba en los Estados Unidos, pubblicata da José Antonio Saco a Parigi nel 1848, contribuì a lanciare un ampio dibattito filosofico e politico. Secondo Saco, il futuro di Cuba avrebbe potuto essere legato unicamente ad una Spagna più liberale e non agli Stati Uniti, poiché «l'annessione, alla fine, non sarebbe una annessione, bensì un assorbimento di Cuba ad opera degli Stati Uniti»47. Questo avrebbe comportato la scomparsa totale del potere creolo, "diluito" all'interno di un paese demograficamente imponente, anglofono e protestante. In un certo senso, Saco voleva evitare per Cuba il destino dello svuotamento culturale che l'Unione impose alla Louisiana francofona (di cui Saco fu diretto testimone nei suoi soggiorni statunitensi)48. Saco, nel suo rifiuto di un'annessione, già aveva intuito il pericolo che gli Stati Uniti avrebbero potuto rappresentare per l'autonomia culturale (ma anche politica) di Cuba. Infatti, si legge nelle Ideas: "Io vorrei che Cuba non solo fosse ricca, colta e potente, ma anche che Cuba fosse cubana e non anglo-americana»49. Saco venne a scontrarsi, a partire dal 1848, con i principali rappresentanti dei club cubani. Oltre ad un fitto rapporto epistolare con Cisneros Betancourt, in cui affiorarono le divergenze sulla questione annessionista, Saco ebbe un veemente scambio di idee a distanza con un altro simpatizzante delle imprese piratesche di López, Cristóbal Madán50.

L'impotenza degli hacendados cubani di fronte alle strategie geopolitiche dell'abolizione della tratta generò una frustrazione che sarebbe durata anche dopo la

\footnotetext{
${ }^{45}$ Cfr. RAMOS, Julio, Divergent Modernities: Culture and Politics in Nineteenth-Century Latin America, Durham, Duke University Press, 2001, p. 152.

${ }^{46}$ Cfr. TORRENTE, Mariano, op. cit., p. 15.

${ }^{47}$ Cfr. SACO, José Antonio, Ideas sobre la incorporación de Cuba en los Estados Unidos, Paris, Impr. de Panckoucke, 1848, p. 2.

48 Cfr. SACO, José Antonio, Origen del movimiento anexionista en Cuba, in SACO, José Antonio, Colección de papeles científicos, históricos, políticos y de otros ramos sobre la Isla de Cuba ya publicados, ya ineditos por Don José Antonio Saco, Paris, Impr. de D’Aubusson \& Kugelmann, 1859, p. 310; Lettera di José Antonio Saco a Tomás Gener, 24 aprile 1832 in FERNÁNDEZ DE CASTRO, José A., SACO, José Antonio, Medio siglo de historia colonial de Cuba, La Habana, Ricardo Veloso, 1923, pp. 40-41.

49 Cfr. SACO, José Antonio, Ideas sobre la incorporación de Cuba en los Estados Unidos, cit., pp. 2-3. Il corsivo è originale. Sul complesso rapporto tra Saco e la società statunitense cfr. OPATRNY, Josef, «José Antonio Saco y los Estados Unidos», in Revista Brasileira do Caribe, vol. 10, 19, 2/2009, pp. 79-104.

$5^{\circ}$ Cfr. MADÁN, Cristóbal, Contestación a un folleto titulado: Ideas sobre la incorporación de Cuba en los Estados Unidos por Don José Antonio Saco, New York, Imprenta de La Verdad, 1849 .
} 
fine delle imprese di filibustering di Narciso López, ma soprattutto determinò una connessione molto intima tra annessionismo e soluzione (conservatrice) al problema della schiavitù ${ }^{1}$. L'intreccio tra l'espansionismo ed il dibattito sullo schiavismo negli Stati Uniti e le prime espressioni di malcontento a Cuba nei confronti del governo spagnolo risulta evidente: alla metà del secolo, l'espansionismo schiavista dei proprietari di piantagioni degli Stati del Sud aveva una relazione di reciprocità di interessi con la saccarocrazia52 cubana, sedotta dall'idea di annessione ${ }^{53}$. La questione schiavista fu il principale catalizzatore del grande interesse politico ed economico nei confronti dell'annessione di Cuba agli Stati Uniti. Dal 1848 anche la Francia aveva nuovamente abolito la schiavitù nelle proprie colonie e il sistema produttivo cubano si trovava in seria difficoltà, stretto tra due potenze anti-schiaviste (Francia e Inghilterra) ed una madrepatria facilmente ricattabile da entrambe. A Cuba la tratta degli schiavi aveva subito un colpo mortale sotto O'Donnell e, dal 1844, l'ingresso di navi negriere si era praticamente azzerato: la politica spagnola sembrava orientata al passo successivo, ovvero l'eliminazione dell'istituto schiavista54. Lo stesso Villaverde, in un articolo del 1849, analizzava in questi termini la situazione isolana: «I cubani [...] subiscono, da una parte, la minaccia di una insurrezione di schiavi e, dall'altra, essi sono alla mercé di truppe licenziose e mercenarie. In queste circostanze, essi si rendono conto che la loro unica speranza di libertà risiede nell'aiuto dall'esterno, risiede in una qualche forza attorno alla quale essi possano raccogliersi, dare voce alle proprie opinioni e sostenere il proprio diritto a partecipare all'amministrazione dello stato»55.

L'annessionismo cubano fu in buona parte il prodotto di un calcolo politico dettato da necessità contingenti. Una lettera di Gaspar Betancourt Cisneros, annessionista e membro del Consejo Cubano (anche se animato da una forma di «annessionismo patriottico»)56, all'amico Saco metteva in luce le motivazioni della sua scelta politica:

${ }^{11}$ Cfr. URBAN, Stanley C., «The Africanization of Cuban Scare, 1853-1855», in The Hispanic American Historical Review, 37, 1/1957, pp. 30-33.

$5^{2}$ Ossia dei proprietari di piantagioni di canna da zucchero che divenivano, in virtù del proprio potere economico una vera e propria aristocrazia. Sul tema: GONÇALVES, Dominique, Les enjeux politiques de la traite négrière à Cuba: 1760-1840, COTTIAS, Myriam, CUNIN, Elisabeth, de ALMEIDA MENDES, António (sous la direction de), Les traites et les esclavages. Perspectives historiques et contemporaines, Paris, Karthala, 2010, pp. 111-124

${ }^{53}$ Cfr. SAIZ PASTOR, Candelaria, "Narciso López y el anexionismo en Cuba», in Anuario de Estudios Americanos, 43/1986, pp. 455-456.

${ }^{54}$ Cfr. AIMES, Hubert H.S., A History of Slavery in Cuba, 1511 to 1868, New York, Putnam's Sons, 1907, pp. 166-169; SACO, José Antonio, La supresión del tráfico de esclavos en la Isla de Cuba, Paris, Impr. de Panckoucke, 1845.

${ }_{55}^{5}$ Cfr. «Cuba», in The United States Magazine and Democratic Review, 25, 135/1849, p. 200.

${ }^{56}$ Cfr. PORTELL VILÁ, Herminio, op. cit., vol. 1 , p. 190. Fernando Portuondo ha invece messo in luce che «l'essere annessionisti a metà del secolo XIX non era una colpa, bensì una forma di patriottismo» poiché l'alternativa sarebbe stata quella di sostenere il governo coloniale e 
«L'annessione, mio caro Saco, non è un ideale, è un calcolo; anzi, è molto di più, è la legge ineludibile della necessità, è il sacro dovere della propria conservazione» 57 . Inoltre, alcuni membri delle associazioni annessioniste erano di idee liberali e potenzialmente favorevoli ad una graduale abolizione della schiavitù ma, dopo le rivolte razziali degli anni Quaranta, ritenevano la Spagna incapace di organizzare il superamento pacifico della pratica dello schiavismo ${ }^{58}$. Nel proclama lanciato da López in corrispondenza dell'ultima spedizione dell'agosto 1851 si coglie effettivamente la conciliazione degli ideali libertari dello "spirito americano" con la necessità di tutelare gli interessi di proprietà e la struttura politico-economica dell’isola: «La schiavitù domestica non è un fenomeno sociale solamente cubano, né è incompatibile con la libertà dei cittadini. La storia antica e moderna ve lo dimostrano e avete accanto a voi l'esempio degli Stati Uniti, dove tre milioni di schiavi non impediscono il fiorire delle istituzioni tra le più liberali del mondo»59.

Tom Chaffin ha messo in dubbio la spiegazione olistica del binomio schiavismo/annessionismo come unico motore delle imprese di filibustering ai danni del dominio coloniale spagnolo a Cuba. Ovviamente, è innegabile che il dibattito sul futuro schiavista e statunitense della colonia cubana fosse un elemento centrale delle spedizioni di López. Tuttavia, esistono una serie di altri elementi, come l'ampio sostegno di alcuni settori economici e congressuali negli Stati del Nord o le simpatie trasversali per le iniziative anti-colonialiste fiorite a Cuba, che mettono in difficoltà tale impostazione.

La ricerca di una diplomazia informale (che si tradusse anche in una iniziativa militare privata, il filibustering) necessitava la presenza di elementi di contatto ed obiettivi comuni e condivisi. Per questo, gli agenti di López ed il Club de la Habana si mossero in diverse direzioni, sia tra la cerchia dei fedelissimi del presidente Polk, sia tra influenti membri della società schiavista del Sud. Volente o nolente, López dovette sposare la causa schiavista ed annessionista, se non altro per conformità agli ideali dei

corrotto della Spagna. Cfr. PORTUONDO, Fernando, «Independencia y anexión en las conspiraciones de 1848-1849", in Cuadernos de Historia Habanera, 44, 1950, p. 144. Portell Vilá, al contrario, ha operato una divisione netta tra i cubani che vedevano nell'annessione un mero tentativo utilitaristico, una possibilità di arricchimento o un mantenimento del proprio status economico-sociale (José Luis Alfonso e Cristóbal Madán) e coloro i quali la concepivano solamente come extrema ratio (Betancourt Cisneros). Cfr. PORTELL VILÁ, Herminio, op. cit., vol. 1, pp. 190-216.

${ }^{57}$ Lettera di Gaspar Betancourt Cisneros a José Antonio Saco, 19 ottobre, 1848, in FERNÁNDEZ DE CASTRO, José A., SACO, José Antonio, op. cit., p. 94.

${ }^{58}$ Cfr. POYO, Gerald E., «Evolution of Cuban Separatist Thought in the Emigré Communities of the United States,1848-1895», in The Hispanic American Historical Review, 66, 3/1986, pp. 485-489.

${ }^{59}$ Cit. in MORALES Y MORALES, Vidal, op. cit., vol. 2, pp. 158-159. 
propri finanziatori. In realtà, se si analizza il vasto sostegno bipartisan e non settario all'idea centrale del Manifest destiny si comprendono a pieno i diversi interessi che ruotavano attorno alla causa della liberazione di Cuba. La felice intuizione linguistica di O’Sullivan permise di raccogliere attorno ad una stessa idea, così come il filibustering verso Cuba, sia il Nord che il Sud ${ }^{60}$. L'atteggiamento altalenante di alcuni membri democratici del Congresso nei confronti di López ed il fatto che la piattaforma democratica non avesse una posizione definita sul tema rappresentava lo specchio della progressiva frammentazione del partito nel corso degli anni Cinquanta dell'Ottocento, soprattutto sul tema dell'espansionismo ${ }^{61}$. Nel contempo, molti democratici del Sud furono attratti dalla possibilità di coniugare l'espansionismo con l'estensione dello schiavismo per mezzo del repubblicanesimo che animava López ed i suoi seguaci. Il filibustering voleva in qualche modo riproporre l'espansionismo jacksoniano, che fu in grado di coniugare i valori repubblicani con l'estensione territoriale dell'istituto della schiavitù: anche nel triennio 1848-1851 alcuni sudisti vennero attratti dalla possibilità di comporre i contrasti tra l'annessione di un nuovo stato schiavista (Cuba) ed il sostegno per i valori liberali delle rivoluzioni europee del $1848^{62}$. In questa prospettiva risulta evidente l'impatto personalistico nel sostegno al filibustering anche in riferimento ai leader degli stati del Sud: se John Quitman, John Cotesworth Pinckney Smith e John Henderson supportarono e finanziarono le spedizioni di López, molti altri, come John Calhoun, David Yulee, Jefferson Davis e Stephen Mallory, non lo fecero pubblicamente, pur condividendo le motivazioni ${ }^{63}$.

Infatti, mentre il profondo Sud, Louisiana e Mississippi in testa, appoggiò con entusiasmo il generale cubano, gli stati più settentrionali si mostrarono maggiormente riluttanti. A New Orleans, ampiamente compromessa nella preparazione delle imprese di filibustering, una buona percentuale dei membri dei club annessionisti cittadini era rappresentata da mercanti e uomini d'affari (25\%), che non avevano proprietà terriere e non possedevano schiavi (55\%), quindi non certo interessati all'espansione della

\footnotetext{
${ }^{60}$ Cfr. JOHANSSEN, Robert W., The Meaning of Manifest Destiny, in HAYES, Sam W. e MORRIS Christopher (a cura di), Manifest Destiny and Empire: American Antebellum Expansionism, College Station, Texas A\&M University Press, 1997, pp. 10-19 ed anche MERK, Frederick, Manifest Destiny and Mission in American History, Cambridge, Harvard University Press, 1995, pp. 209-210.

${ }^{61}$ Cfr. HIETALA, Thomas R., Manifest Design. American Exceptionalism and Empire, Ithaca, Cornell University Press, 2002, pp. 215-254; HAYNES, Sam W., James K. Polk and the expansionist impulse. New York, Longman Press., 2002, pp. 117-118, 140.

${ }^{62}$ Cfr. CHAFFIN, Tom, op. cit., pp. 8-10.

${ }^{63}$ Ibidem, pp. 82-85.
} 
schiavitù verso i Caraibi64. Senza contare che anche gli Stati del Nord avevano un certo interesse alla liberazione di Cuba dalla Spagna (seguita da una eventuale annessione). Ad esempio, la United States Mail Steamship Company, fondata nel 1848, che aveva ottenuto l'appalto per la consegna della corrispondenza tra New York e la California (via New Orleans, La Habana e Panamá), fu accusata dalle autorità spagnole di imbarcare a bordo delle proprie navi anche armi destinate ai filibuster, libri ed articoli di propaganda anti-spagnola ed emissari di López in visita a Cuba65. Inoltre, il principale organo di stampa a supporto dell'annessionismo, il periodico «La Verdad», veniva stampato proprio a New York. La diplomazia informale del filibustering, così come all'interno del dibattito sull'annessione del Texas e sulla Guerra al Messico, creò un movimento a sostegno di López che travalicava i confini e gli interessi regionalisti degli Stati Uniti. A questo proposito, Robert May ha messo in luce come l'espansionismo sudista e lo schiavismo fossero il motivo dominante delle imprese di flibustering solamente dopo il Kansas-Nebraska Act del 1854, ovvero quando, in riferimento a Cuba, gli annessionisti cubano-americani contattarono John Quitman, ex Governatore del Mississippi, per invadere nuovamente l'isola. Dalla metà degli anni Cinquanta, furono i proprietari delle piantagioni del Sud a prendere in mano le redini delle operazioni verso Cuba (anche in considerazione degli attriti con alcuni membri della comunità cubano-americana) in corrispondenza della reale minaccia di una imminente abolizione della schiavitù sull'isola ${ }^{66}$.

In conclusione, l'esperienza del filibustering di López strutturò una rete di relazioni che sarebbe sopravvissuta alla sua morte. Alcuni partecipanti alle spedizioni del 18491851 raccolsero, almeno per un lustro, l'eredità dell'esperienza cubano-americana per riproporre imprese del tutto simili: Roberdeau Wheat, Theodore O'Hara, Louis Schlessinger, Achilles Kewen e Callender Fayssoux parteciparono alle iniziative militari di William Walker in Nicaragua, mentre i quadri organizzativi cubano-americani continuarono i propri sforzi per progettare un'invasione dell'isola ${ }^{67}$. Il fallimento delle imprese di invasione di Cuba nel triennio 1849-1851 misero in evidenza tutti i limiti della diplomazia (e del warfare) privata. Nel corso delle due spedizioni che raggiunsero l'isola (quella del 1850 e l'ultima del 1851), López e compagni mal risposero la propria fiducia nella volontà dei cubani di insorgere contro la dominazione spagnola e vennero

\footnotetext{
${ }^{64}$ Cfr. TANSEY, Richard, op. cit., p. 249.

${ }^{65}$ Cfr. CHAFFIN, Tom, op. cit., p. 175.

${ }^{66}$ Cfr. MAY, Robert E., "Young American Males and Filibustering in the Age of Manifest Destiny: The United States Army as a Cultural Mirror», cit., pp. 873-882; MAY, Robert E., The Southern Dream of a Caribbean Empire, 1854-1861, Gainesville, University Press of Florida, 2002, pp. 10-11, 19-21, 48-76.

${ }^{67}$ Cfr. MAY, Robert E., The Southern Dream of a Caribbean Empire, 1854-1861, pp. 29-30.
} 
chiaramente tratti in inganno dalle informazioni che giungevano dall'isola. Soprattutto nel 1851, l'ultima spedizione venne organizzata sulla spinta della sollevazione di Joaquín Agüero y Agüero che, tuttavia, venne abortita sul nascere, lasciando gli invasori cubano-americani al loro destino. Nella sua ultima lettera alla famiglia prima dell'esecuzione, William L. Crittenden, illustre figlio dell'aristocrazia americana e membro della spedizione del 1851, scrisse che López attese invano rinforzi improbabili ed una sollevazione popolare impossibile ${ }^{68}$. La strategia militare mostrò le sue incredibili carenze, soprattutto negli armamenti, e la forza numerica degli invasori fu sovrastimata. Come ha sottolineato Quisenberry, sarebbe stato sufficiente un piccolo contingente navale aggiuntivo, di cui ovviamente l'operazione privata non disponeva, per conquistare La Habana, lasciata completamente senza difese a causa delle operazione anti-pirateria dell'esercito coloniale ${ }^{69}$. Inoltre, la diplomazia informale cubano-americana aveva radunato uomini di estrazione sociale e nazionalità differenti, ma, soprattutto, alla testa di questo variegato "esercito" vi era un generale che non parlava la stessa lingua dei propri soldati, che per lo più erano americani (López non conosceva l'inglese) ${ }^{70}$.

L'epopea del filibustering di López, che contribuì anche iconicamente alla causa della separazione di Cuba dalla Spagna ${ }^{71}$, ebbe anche il merito di rilanciare il dibattito interno all'esilio cubano negli Stati Uniti e sull'isola proprio sulle questioni centrali (annessionismo, indipendenza, schiavismo, strategia di lotta per la liberazione e cubanidad) che permisero la strutturazione delle imprese di filibustering di metà Ottocento, rafforzando ed approfondendo quei legami people-to-pleople tra i due paesi che sarebbero proseguiti sino a giorni nostri. In modo particolare, gli anni Quaranta dell'Ottocento contribuirono a formulare un'immagine politica, economica e sociale di Cuba che, per quanto distorta, avrebbe giocato un ruolo fondamentale nella lotta per l'indipendenza ${ }^{72}$. Infine, l'esecuzione di alcuni americani della spedizione del 1851, in

\footnotetext{
${ }^{68}$ Cit. in QUISENBERRY, Anderson C., op. cit., pp. 89-90.

${ }^{69}$ Ibidem, pp. 117-118

${ }^{70}$ Cfr. CALDWELL, Robert G., op. cit., p. 92.

${ }^{71}$ La vessillo che López e i suoi uomini utilizzarono per le spedizioni, mutuato da quella della Repubblica texana del 1836, divenne quella ufficiale della Repubblica di Cuba del 1902. Cfr. PORTELL VILÁ, Herminio, op. cit., vol. 1, pp. 273-277.

${ }_{72}$ Circa il misunderstanging culturale e politico tra Stati Uniti e Cuba nella seconda metà dell'Ottocento cfr. PÉREZ JR., Louis A., Cuba in the American Immagination: Metaphor and the Imperial Ethos, Chapel Hill, University of North Carolina Press, 2008; IGLESIAS, Marial U., Imágenes en conflicto: Cuba y la presencia imperial norteamericana (1898-1902), in LORINI, Alessandra (edited by), An Intimate and Contested Relation: The United States and Cuba in the Late Nineteenth and Early Twentieth Centuries, Firenze, Firenze University Press, 2005, pp. 31-53; LORINI, Alessandra, L'impero della libertà e l'isola strategica: gli Stati Uniti e Cuba tra Otto e Novecento, Napoli, Liguori, 2007, capp. 1-2. Sul problema della schiavitù nella lotta per
} 
particolare William Crittenden, avrebbe profondamente scosso ed indignato l'opinione pubblica statunitense, alla stessa stregua dell'affondamento del Maine nel 1898, casus belli per l'intervento americano contro la Spagna, fomentando la leyenda negra antispagnola, ampiamente utilizzata sia dal nativismo sia dal yellow journalism negli anni precedenti la Guerra Ispano-Americana73. Con López, infatti, nacque un considerevole interesse sociale e politico nei confronti dei movimenti indipendentisti isolani che, alla fine del secolo, avrebbe contribuito all'intervento americano nella guerra contro la Spagna, facendo precipitare Cuba in quello che Louis A. Pérez Jr. ha definito «debito di gratitudine» nei confronti degli statunitensi e che avrebbe segnato le relazioni cubanoamericane anche dopo la Rivoluzione castrista del $1959^{74}$.

l'indipendenza cubana cfr. HELG, Aline, Our Rightful Share: The Afro-Cuban Struggle for Equality, 1886-1912, Chapel Hill, University of North Carolina Press, 1995.

${ }_{73}$ QUISENBERRY, Anderson C., op. cit., p. 95; Circa il pregiudizio anti-ispanico cfr. Wayne Powell Philip, The Tree of Hate: Propaganda and Prejudices Affecting United States Relations with the Hispanic World, New York, Basic Books, 1971 ed anche WESTON, Rubin F., Racism in U.S. Imperialism: the Influence of Racial Assumptions on American Foreign Policy, 1893-1946, Columbia, University of South Carolina Press, 1989.

${ }^{74}$ Cfr. PÉREZ JR., Louis A., «Incurring a Debt of Gratitude: 1898 and the Moral Sources of United States Hegemony in Cuba», in The American Historical Review, 104, 2/1999, pp. 356398. Sull'epopea di López, interpretata dalla Rivoluzione castrista come primo esempio dell'ingerenza militare americana nelle vicende interne cubane, cfr RAMONET, Ignacio, Fidel Castro: My Life. A Spoken Autobiography, New York, Scribner Book Company, 2007, pp. 143145 . 


\section{* L'autore}

Alessandro Badella è dottorando in Scienze Politiche presso l'Università degli Studi di Genova con un progetto sul ruolo del sistema internazionale nella transizione post-socialista di Cuba. Nel 2012 ha pubblicato il volume monografico La spina nel fianco. Stati Uniti e Cuba in una prospettiva razziale, 1823-1912 (Salerno, Arcoiris). Collabora con diversi think tank geopolitici, tra cui ISPI, per i quali scrive articoli sulle relazioni tra Cuba e Stati Uniti.

URL: < http://www.studistorici.com/progett/autori/\#Badella >

\section{Per citare questo articolo:}

BADELLA, Alessandro, «Unlawful enterprise. II filibustering di Narciso López: people-to-people diplomacy tra schiavismo ed annessionismo», Diacronie. Studi di Storia Contemporanea: Contrabbandieri, pirati e frontiere: per una storia delle pratiche informali nell'America Centrale (XVII-XXI secolo), 29/04/2013,

URL:<http://www.studistorici.com/2013/04/29/badella_numero_13/ >

\section{Diacronie Studi di Storia Contemporanea $\beta$ www.diacronie.it}

Risorsa digitale indipendente a carattere storiografico. Uscita trimestrale. redazione.diacronie@hotmail.it

Comitato di redazione: Marco Abram - Jacopo Bassi - Luca Bufarale - Alessandro Cattunar - Elisa Grandi - Deborah Paci - Fausto Pietrancosta - Matteo Tomasoni - Luca Zuccolo

Diritti: gli articoli di Diacronie. Studi di Storia Contemporanea sono pubblicati sotto licenza Creative Commons 2.5 Possono essere riprodotti a patto di non modificarne i contenuti e di non usarli per fini commerciali. La citazione di estratti è comunque sempre autorizzata, nei limiti previsti dalla legge. 